\title{
OPEN Identifying the major lactate transporter of Toxoplasma gondii tachyzoites
}

\author{
Joy M. Zeng ${ }^{1}$, Sanduni V. Hapuarachchi ${ }^{1}$, Sarah H. Shafik ${ }^{1}$, Rowena E. Martin ${ }^{1}$, Kiaran Kirk ${ }^{1}$,
} Giel G. van Dooren ${ }^{1,2 \otimes}$ \& Adele M. Lehane ${ }^{1,2 \otimes}$

Toxoplasma gondii and Plasmodium falciparum parasites both extrude L-lactate, a byproduct of glycolysis. The $P$. falciparum Formate Nitrite Transporter, PfFNT, mediates L-lactate transport across the plasma membrane of $P$. falciparum parasites and has been validated as a drug target. The $T$. gondii genome encodes three FNTs that have been shown to transport L-lactate, and which are proposed to be the targets of several inhibitors of $T$. gondii proliferation. Here, we show that each of the TgFNTs localize to the $T$. gondii plasma membrane and are capable of transporting L-lactate across it, with TgFNT1 making the primary contribution to L-lactate transport during the disease-causing lytic cycle of the parasite. We use the Xenopus oocyte expression system to provide direct measurements of L-lactate transport via TgFNT1. We undertake a genetic analysis of the importance of the tgfnt genes for parasite proliferation, and demonstrate that all three tgfnt genes can be disrupted individually and together without affecting the lytic cycle under in vitro culture conditions. Together, our experiments identify the major lactate transporter in the disease causing stage of $T$. gondii, and reveal that this transporter is not required for parasite proliferation, indicating that $T g$ FNTs are unlikely to be targets for anti-Toxoplasma drugs.

Toxoplasma gondii and Plasmodium falciparum are unicellular protozoan parasites that belong to the phylum Apicomplexa. P. falciparum is the most deadly of the Plasmodium species that cause malaria in humans ${ }^{1}$. T. gondii infects a large proportion of the world's population and can cause severe disease in immunocompromised individuals ${ }^{2}$. T. gondii can also have devastating effects on the development of foetuses when it infects women during pregnancy ${ }^{2}$. New medicines are needed urgently against both parasites.

The disease-causing tachyzoite stage of T. gondii parasites utilizes both glucose and glutamine as energy sources for the generation of $\mathrm{ATP}^{3,4}$. These parasites acquire glucose through a plasma-membrane localized Glucose Transporter $(\mathrm{TgGT} 1)^{3}$. Glucose is metabolized via glycolysis to generate pyruvate. Pyruvate has multiple possible fates in the parasite ${ }^{5}$. It can be transported into the mitochondrion, where it is further metabolized by the TCA cycle to generate ATP by oxidative phosphorylation ${ }^{6}$. Alternatively, pyruvate can be metabolized to form L-lactate, which is then exported from the parasite $\mathrm{f}^{7,8}$.

Lactate synthesis is catalyzed by lactate dehydrogenase (LDH). The T. gondii genome encodes two LDH enzymes ( $T g \mathrm{LDH} 1$ and $T g \mathrm{LDH} 2)$, neither of which is required for proliferation of the disease-causing tachyzoite stage under standard in vitro culture conditions ${ }^{9,10}$. T. gondii tachyzoites that are rendered defective in glucose import or some aspects of glycolysis can still grow in vitro and in vivo, catabolizing glutamine via the TCA cycle to produce ATP and generating essential glycolytic intermediates via gluconeogenesis ${ }^{4,711-13}$. When glucose and glutamine are both available, both are used for ATP generation ${ }^{7}$.

By contrast, the disease-causing blood stages of $P$. falciparum parasites rely almost exclusively on glycolysis to generate $\mathrm{ATP}^{14}$. Here, $\mathrm{L}$-lactate is the major product of glycolysis and must be exported from the parasite in symport with $\mathrm{H}^{+}$by the P. falciparum Formate Nitrite Transporter (PfFNT) present on the parasite's plasma membrane ${ }^{15,16}$. Members of the FNT family transport a variety of monocarboxylates and other anions ${ }^{17-19}$. They are found in numerous prokaryotic and eukaryotic microorganisms, but are not present in mammalian cells ${ }^{15}$. Compounds that kill $P$. falciparum parasites via inhibition of $P f F N T$ have recently been identified, thereby validating PfFNT as a novel antimalarial drug target ${ }^{20,21}$.

A recent study provided evidence that the three homologues of PfFNT encoded in the T. gondii genome are L-lactate transporters that localize to the plasma membrane when expressed from a non-native promoter ${ }^{22}$.

${ }^{1}$ Research School of Biology, Australian National University, Canberra, ACT 2601, Australia. ${ }^{2}$ These authors jointly supervised this work: Giel G. van Dooren and Adele M. Lehane. ${ }^{\circledR}$ email: giel.vandooren@anu.edu.au; adele.lehane@ anu.edu.au 


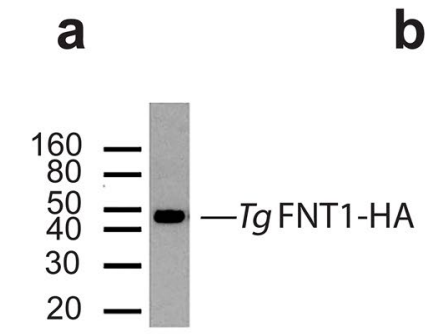

b



Plasma
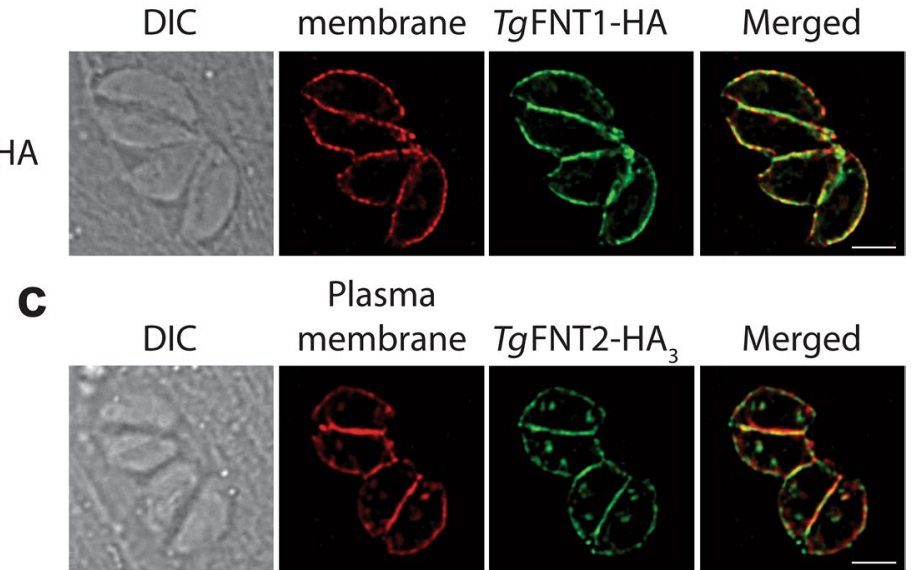

Plasma
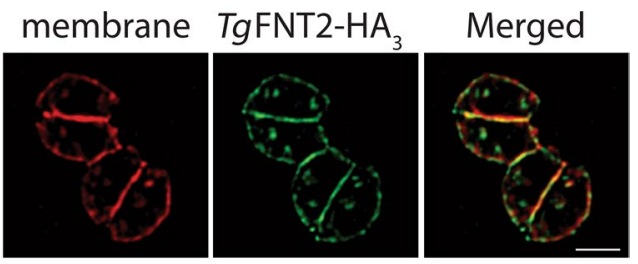

d
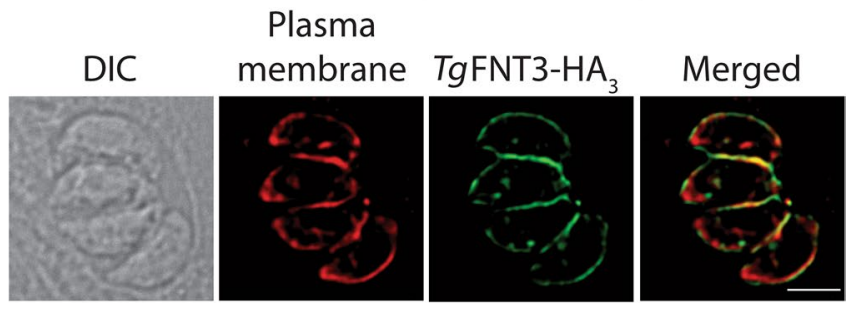

Figure 1. $T g$ FNT1 and ectopically-expressed $T g F N T 2$ and $T g F N T 3$ localize to the plasma membrane of T. gondii. (a) Western blot of TgFNT1-HA-expressing parasites performed using an anti-HA antibody. The uncropped image is shown in Supplementary Fig. S3. (b-d) Immunofluorescence assay reveals co-localization of TgFNT1-HA (b; green), $T g F N T 2-\mathrm{HA}_{3}$ (c; green) and $T g \mathrm{FNT}_{3}-\mathrm{HA}_{3}$ (d; green) with the plasma membrane marker $\mathrm{P} 30^{39}$ (red). The scale bars represent $2 \mu \mathrm{m}$.

Compounds found to inhibit the activity of the transporters showed some inhibition of tachyzoite proliferation at micromolar concentrations, leading to the suggestion that the TgFNTs might be essential during the T. gondii lytic cycle and 'druggable'22. The possibility that the compounds inhibited parasite growth via alternate means ('off-target effects') was not excluded. Although T. gondii tachyzoites can survive without glucose, it is possible that in the presence of glucose, the lack of a L-lactate efflux mechanism would cause L-lactate to accumulate intracellularly to concentrations sufficient to jeopardize $\mathrm{pH}$ regulation and/or osmotic stability. However, a genome-wide screen has provided evidence that each of the TgFNTs are dispensable during the lytic cycle ${ }^{23}$, and a TgFNT1 knockout parasite line was recently generated and found to grow normally despite secreting less lactate into the medium than wild-type parasite ${ }^{24}$. Here, we employed genetic and physiological approaches to investigate the roles of the three $\mathrm{TgFNTs}$ in situ and to determine whether the activity of one or more $T g \mathrm{FNTs}$ is required for the proliferation of $T$. gondii tachyzoites.

\section{Results}

TgFNTs localize to the parasite plasma membrane. The T. gondii genome encodes three members of the FNT family, termed TgFNT1 (TGGT1_209800), TgFNT2 (TGGT1_292110) and TgFNT3(TGGT1_229170)22. The TgFNTs share considerable sequence similarity with each other, with PfFNT, and with the well-characterized E. coli FNT family member FocA (Supplementary Fig. S1).

To investigate the expression and localization of each TgFNT, we attempted to generate parasite lines in which a single hemagglutinin (HA) tag was fused to the $3^{\prime}$ end of the open reading frame of the encoding gene. This approach was successful for TgFNT1 (Supplementary Fig. S2). Western blotting with an anti-HA antibody revealed that the resultant $\mathrm{TgFNT} 1-\mathrm{HA}$ protein is expressed in tachyzoites and that it had the expected mass of approximately $45 \mathrm{kDa}$ (Fig. 1a). Immunofluorescence assays revealed that $T g \mathrm{FNT} 1-\mathrm{HA}$ localizes to the $T$. gondii plasma membrane (Fig. 1b).

Our attempts to generate parasites expressing detectable levels of TgFNT2-HA or TgFNT3-HA with this approach were not successful. An alternate approach was therefore used to localize TgFNT2 and TgFNT3. Parasites were transiently transfected with a vector containing the open reading frame of the encoding gene fused at the $3^{\prime}$ end to a $3 \times \mathrm{HA}$ tag, under the regulation of the constitutive $\alpha$-tubulin promoter. Consistent with the findings of Erler et al. ${ }^{22}$, the ectopically expressed $\mathrm{TgFNT} 2-\mathrm{HA}_{3}$ and $\mathrm{TgFNT} 3-\mathrm{HA}_{3}$ proteins both localized to the T. gondii plasma membrane (Fig. 1c,d).

TgFNTs are not required for tachyzoite proliferation in vitro. To determine whether any of the TgFNTs are important for parasite proliferation, we used CRISPR-Cas9 targeted genome editing to generate 

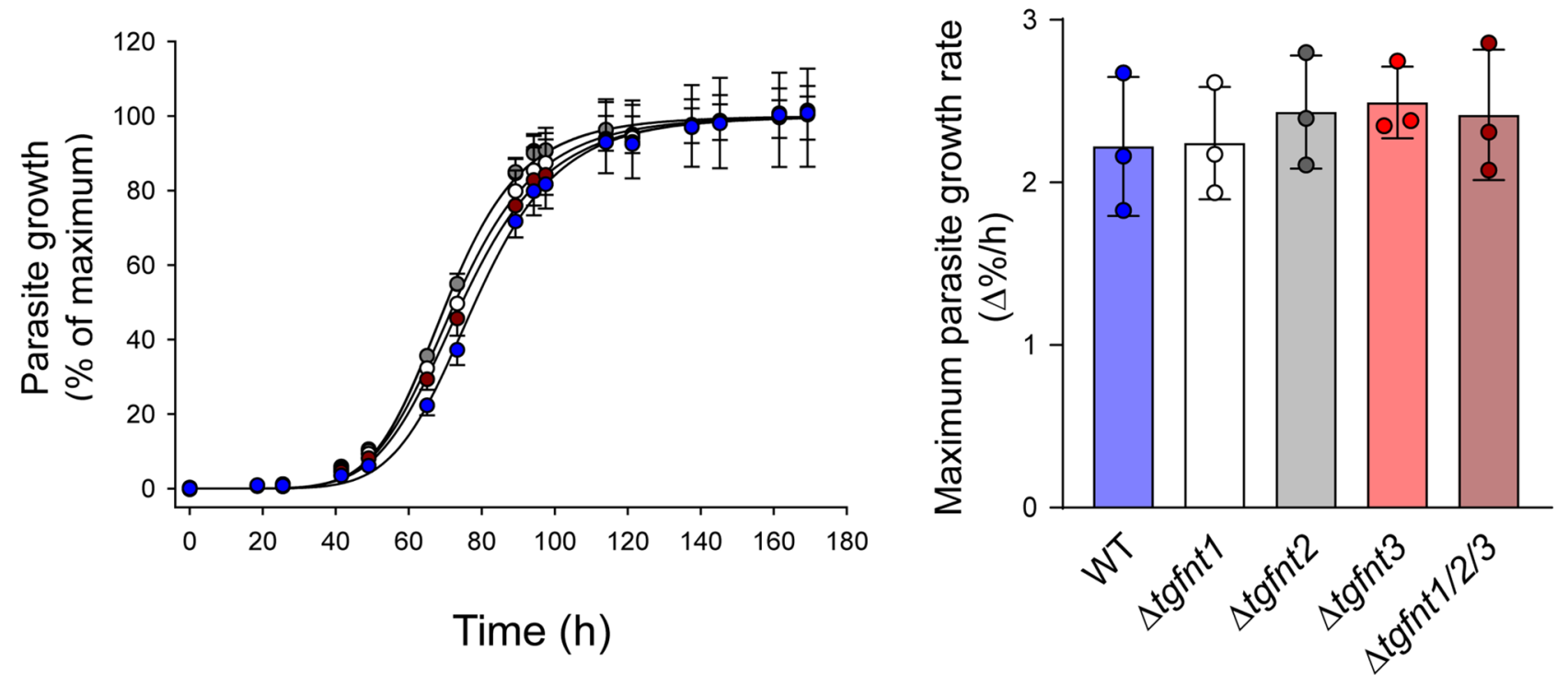

Figure 2. $\mathrm{TgFNT}$ are not required for tachyzoite proliferation in vitro. (a) Data from a single representative experiment showing the growth of the different parasite lines (wild-type, blue; $\Delta t g f n t 1$, white; $\Delta t g f n t 2$, grey; $\Delta \operatorname{tg} f n t 3$, red; $\Delta \operatorname{tg} f n t 1 / 2 / 3$, dark red). Parasite growth was normalized to the maximum level reached for each parasite line after subtraction of the background fluorescence. The mean and SD from triplicate measurements shown. Where not shown, error bars fall within the symbols. For clarity, only positive or negative error bars are shown for the data for certain parasite lines. (b) The maximum growth rate for each parasite line. The bars show the mean \pm SD obtained from three independent experiments for each line, and the symbols show the results obtained in individual experiments. There was no significant difference in the maximum growth rate between any of the parasite lines ( $P=0.8$; one-way ANOVA).

parasites in which the open reading frame of the tgfnt1, tgfnt2 or tgfnt 3 gene was disrupted by frameshift mutations. For $\operatorname{tg} f n t 1$, we selected a clone (' $\Delta t g f n t 1$ ') with a single nucleotide insertion at position 176 from the start

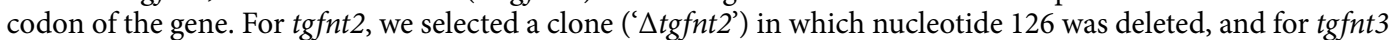
(' $\Delta t g f n t 3$ ') we selected a clone with an insertion of five nucleotides from position 123 of the gene. In all cases, the resulting frameshifts led to the introduction of premature stop codons and truncation of the encoded proteins (Supplementary Fig. S4), which are likely to render the resulting proteins non-functional. We also generated a parasite line in which all three tgfnt genes were disrupted. This was performed by first targeting the tgfnt 1 gene in $\Delta t g f n t 3$ parasites. We selected a clone (' $\Delta \operatorname{tg} f n t 1 / 3$ ') that had a single nucleotide insertion at position 176 of the $\operatorname{tg} f n t 1$ gene. Next, we targeted the $\operatorname{tgfnt} 2$ gene in $\Delta \operatorname{tg} f n t 1 / 3$ parasites. We selected a clone (' $\left.\Delta \operatorname{tg} f n t 1 / 2 / 3^{\prime}\right)$ with a single nucleotide insertion at position 127 of the tgfnt 2 gene. Each of the tgfnt genes in $\Delta \operatorname{tg} f n t 1 / 2 / 3$ parasites has a premature stop codon (Supplementary Fig. S4).

We tested whether any of the $\mathrm{TgFNT}$ isoforms were important for parasite proliferation. We first assessed this using plaque assays. Plaques (zones of clearance) in a host cell monolayer result from multiple cycles of host cell invasion, replication and lysis, with the size of the plaques providing an indication of parasite growth rate. $\Delta \operatorname{tgfnt1}, \Delta \operatorname{tgfnt2}, \Delta \operatorname{tg} f n t 3$, and $\Delta \operatorname{tg} f n t 1 / 2 / 3$ parasites all produced plaques comparable in size to those of their parents (Supplementary Fig. S5), indicating no significant growth defect in any of the four knockout lines.

We also compared the growth rates of the different parasites using a fluorescence assay (described previously ${ }^{25}$ ), taking advantage of the fact that all the parasites express a tandem dimeric Tomato (tdTomato) red fluorescent protein. In each experiment, a sigmoidal growth curve was generated for each parasite line, with a lag phase, exponential growth phase and plateau (Fig. 2a). Using the maximum slope of the growth curve as

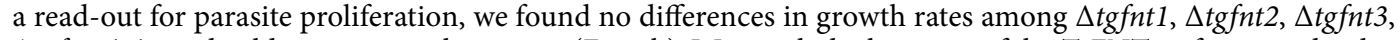
$\Delta \operatorname{tg} f n t 1 / 2 / 3$, and wild-type parental parasites (Fig. 2b). We conclude that none of the TgFNT isoforms, individually or collectively, are required for the normal proliferation of tachyzoites under in vitro culture conditions.

TgFNTs transport L-lactate, with TgFNT1 making the primary contribution to L-lactate transport across the plasma membrane in extracellular tachyzoites. We used the tgfnt knockout parasites to investigate whether one or more of the TgFNTs provide a route for L-lactate transport across the plasma membrane in T. gondii. The parasite experiments described in this section were all performed with extracellular tachyzoites that had either recently emerged from their host cells or that had been manually released from their host cells by passage of the cell culture through a $26 \mathrm{G}$ needle. First, we investigated the effect on parasite cytosolic $\mathrm{pH}$ of adding a high concentration of L-lactate to the extracellular solution. This approach has been used previously with $P$. falciparum parasites to investigate PfFNT, which transports L-lactate in symport with $\mathrm{H}^{+}$ions ${ }^{15,16}$. 

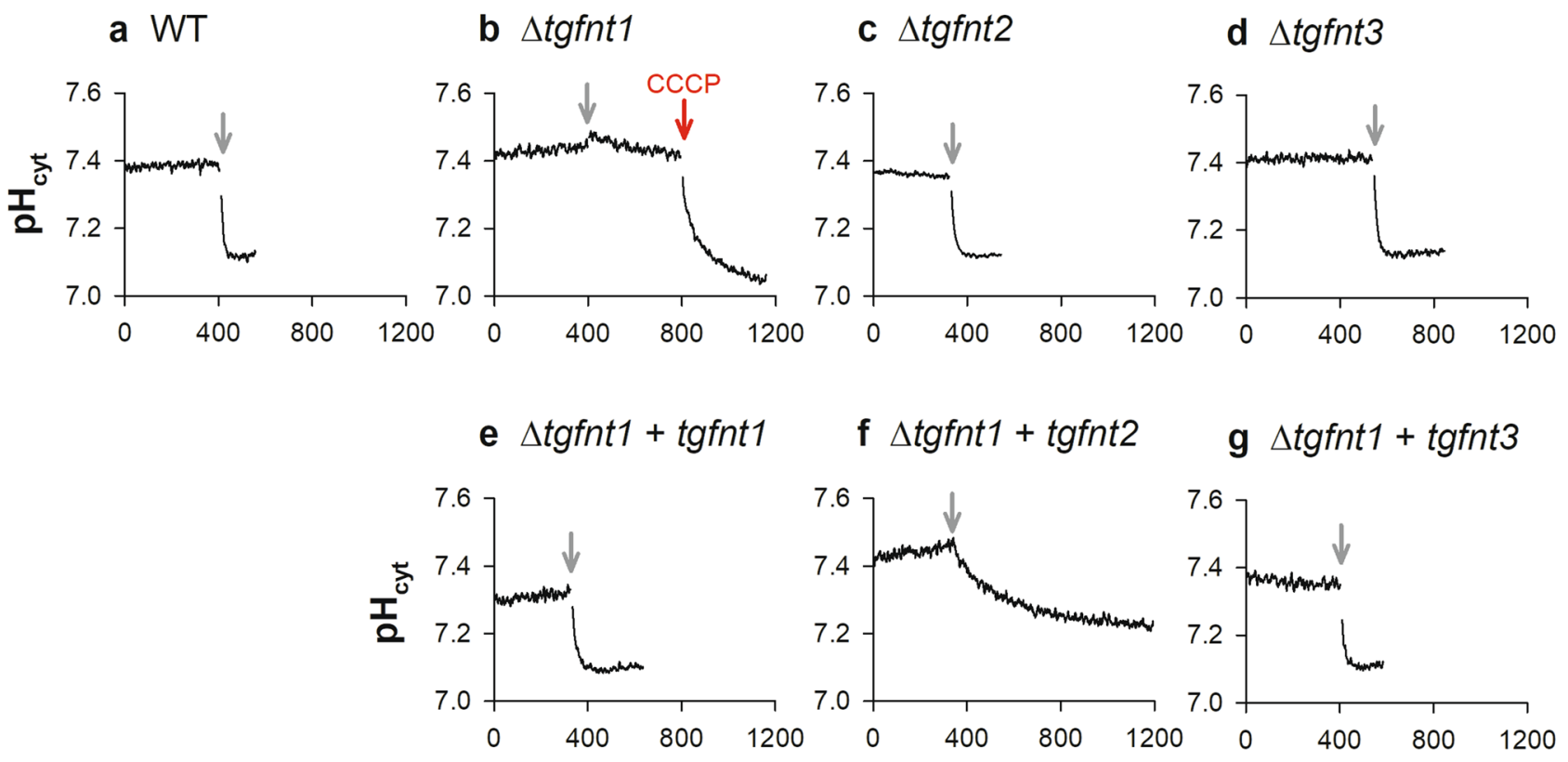

Time (s)

Figure 3. Measurements of the effect of $\mathrm{L}$-lactate on cytosolic $\mathrm{pH}\left(\mathrm{pH}_{\mathrm{cyt}}\right)$ reveal a role for $\mathrm{TgFNT} 1 \mathrm{in}$ lactate transport across the parasite plasma membrane. Extracellular tachyzoites were loaded with BCECF and suspended in Saline Solution at $4{ }^{\circ} \mathrm{C}$. The grey arrows denote the addition of $10 \mathrm{mM} \mathrm{L}$-lactate. The red arrow denotes the addition of the protonophore CCCP $(10 \mu \mathrm{M})$. The top panels show data for $\Delta \operatorname{tgfnt} 1(\mathbf{b}), \Delta \operatorname{tgfnt} 2$ (c), $\Delta \operatorname{tgfnt3}$ (d) parasites and their wild-type parental parasites (a). The bottom panels show data for $\Delta \operatorname{tg} f n t 1$ parasites complemented with tgfnt1 (e), tgfnt2 (f) or tgfnt3 $(\mathbf{g})$. The traces are from a single experiment for each parasite line, and are representative of those obtained in at least three independent experiments. The time taken to reach a minimum $\mathrm{pH}_{\text {cyt }}$ value after the addition of L-lactate was $>400 \mathrm{~s}$ in all experiments for $\Delta \operatorname{tgfnt} 1$ parasites complemented with tgfnt 2 , compared to $<150 \mathrm{~s}$ in all experiments for WT parasites, $\Delta \operatorname{tgfnt} 2$ parasites, $\Delta t g f n t 3$ parasites, and $\Delta \operatorname{tgfnt1}$ parasites complemented with $\operatorname{tg} f$ tnt 1 or $\operatorname{tg} f n t 3$.

Although the physiological role of PfFNT is to remove L-lactate from the parasite cytosol, it is bidirectional. Thus, when a high concentration of L-lactate is added to the extracellular solution to impose an inward L-lactate concentration gradient, a decrease in cytosolic $\mathrm{pH}$ resulting from $\mathrm{L}$-lactate: $\mathrm{H}^{+}$entry can be detected ${ }^{15,21}$.

We measured cytosolic $\mathrm{pH}$ in extracellular T. gondii tachyzoites by loading them with the $\mathrm{pH}$-sensitive dye $\mathrm{BCECF}$. The measurements were performed at $4{ }^{\circ} \mathrm{C}$ to reduce the rate at which $\mathrm{pH}$-regulatory mechanism(s) (including the $\mathrm{H}^{+}$-extruding V-type $\mathrm{H}^{+} \mathrm{ATPase}^{26,27}$ ) counteract the L-lactate-mediated $\mathrm{pH}$ change. When the wild-type parental parasites suspended in a physiological saline solution were exposed to $10 \mathrm{mM} \mathrm{L-lactate,} \mathrm{we}$ observed a rapid decrease in the $\mathrm{pH}$ of the parasite cytosol (Fig. 3a). An abrupt L-lactate-induced decrease in cytosolic $\mathrm{pH}$ was also seen in $\Delta \operatorname{tg} f n t 2$ (Fig. 3c) and $\Delta \operatorname{tgfnt3}$ (Fig. 3d) parasites. In contrast, the addition of $10 \mathrm{mM}$ L-lactate to $\Delta t g f n t 1$ parasites had little effect on the $\mathrm{pH}$ of the cytosol (Fig. 3b), consistent with parasites lacking $T g$ FNT1 having a greatly reduced capacity for $\mathrm{L}-$ lactate: $\mathrm{H}^{+}$symport across the plasma membrane. The subsequent addition of the protonophore CCCP $(10 \mu \mathrm{M})$ dissipated the $\mathrm{H}^{+}$gradient across the plasma membrane, with the cytosolic $\mathrm{pH}$ decreasing to a value close to the extracellular $\mathrm{pH}$ (Fig. 3b), demonstrating the responsiveness of the $\mathrm{pH}$-detection system in these cells.

To confirm that the reduction in L-lactate: $\mathrm{H}^{+}$transport in $\Delta t g f n t 1$ parasites was a consequence of the absence of $T g$ FNT1 expression, we complemented $\Delta \operatorname{tg} f n t 1$ parasites with an ectopically-expressed copy of $\operatorname{tg} f n t 1$ (under the control of the constitutive $\alpha$-tubulin promoter). In these parasites, the addition of $10 \mathrm{mM} \mathrm{L}$-lactate to the extracellular solution caused a pronounced decrease in cytosolic $\mathrm{pH}$ (Fig. 3e). These data are consistent with $T g$ FNT1 serving as the primary L-lactate: $\mathrm{H}^{+}$transporter on the plasma membrane in extracellular tachyzoites.

We also investigated whether $T g$ FNT2 or $T g F N T 3$ could restore L-lactate: $\mathrm{H}^{+}$transport in $\Delta$ tgfnt1 parasites when constitutively expressed in these parasites. We found that $\Delta \operatorname{tg} f n t 1$ parasites complemented with either $\operatorname{tg} f n t 2$ or $\operatorname{tg} f n t 3$ under the control of the a-tubulin promoter underwent a decrease in cytosolic $\mathrm{pH}$ on addition of $10 \mathrm{mM}$ L-lactate to the extracellular solution (Fig. 3f,g). The rate of the $\mathrm{L}$-lactate -induced cytosolic $\mathrm{pH}$ decrease was lower in $\Delta \operatorname{tgfnt1}$ parasites complemented with tgfnt2 (Fig. 3f) than in parasites complemented with tgfnt1 (Fig. 3e) or tgfnt3 (Fig. 3g). The initial rates $(\Delta \mathrm{pH} /$ min, mean $\pm \mathrm{SEM}, \mathrm{n}=3$; determined by fitting lines to the initial linear portions of the traces) were $0.82 \pm 0.19,0.18 \pm 0.09$, and $0.57 \pm 0.10$ for $\Delta \operatorname{tg} f n t 1$ parasites complemented with $\operatorname{tg} f n t 1, \operatorname{tg} f n t 2$ and $\operatorname{tg} f n t 3$, respectively. There was a significant difference between the rates of $\mathrm{pH}$ decrease for $\Delta t g f n t 1$ parasites complemented with $\operatorname{tgfnt} 2$ and $\Delta \operatorname{tgfnt} 1$ parasites complemented with either $\operatorname{tgfnt} 1$ or tgfnt3 $(P<0.05$, unpaired t-tests). This could result from a difference between $T g$ FNT2 and the other $T g$ FNTs with respect to transporter function, regulation, and/or plasma membrane expression. Together, our data show 


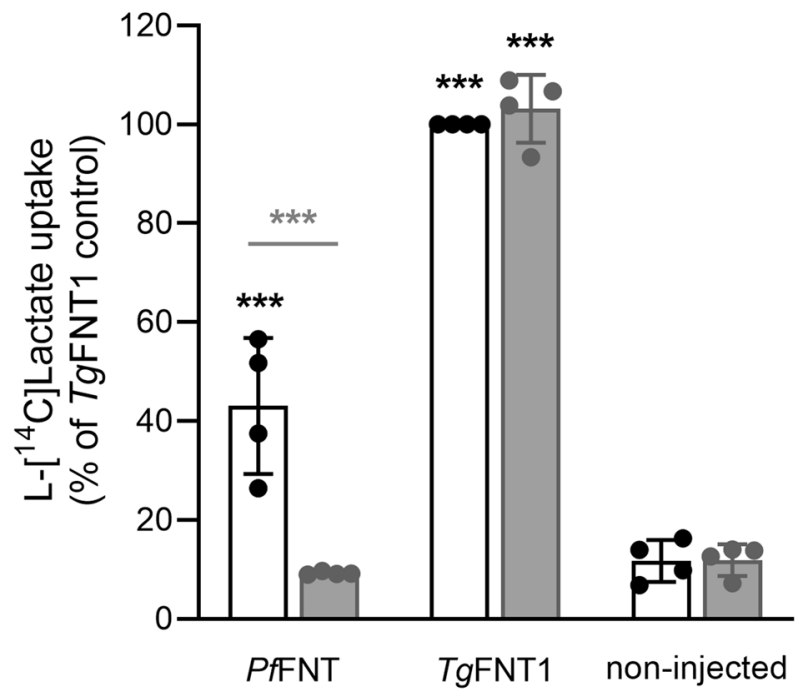

Figure 4. TgFNT1 transports L-lactate in Xenopus oocytes. $\mathrm{L}-\left[{ }^{14} \mathrm{C}\right]$ lactate uptake into non-injected oocytes and oocytes expressing PfFNT or TgFNT1-HA was measured in the absence of compound (0.008\% v/v DMSO; solvent control; white bars) and in the presence of the PfFNT inhibitor MMV007839 ( $4 \mu \mathrm{M}$; grey bars). The uptake of $\mathrm{L}-\left[{ }^{14} \mathrm{C}\right]$ lactate is expressed as a percentage of that determined for $\mathrm{TgFNT}$ F-HA in the absence of MMV007839. The data are averaged from four independent experiments (using oocytes from different frogs), within which measurements were made from 7 to 10 oocytes per treatment. The symbols show the data from each experiment and the bars show the mean \pm SD. Where not shown, error bars fall within the symbols. The data were tested for statistical difference from the non-injected control (black asterisks). The data obtained in the presence or absence of MMV007839 were also compared for each oocyte type (grey asterisks). ${ }^{* \star *} P<0.001$ (one-way ANOVA with post hoc Tukey test); other comparisons did not show significant differences.

that knockout of $\mathrm{TgFNT} 1$ prevents L-lactate -induced $\mathrm{pH}$ changes in the parasite cytosol, which can be rescued by ectopic expression of $T g F N T 1, T g F N T 2$ and $T g F N T 3$. Our data also indicate that $T g F N T 2$ and $T g$ FNT3 make minimal contribution to $\mathrm{L}$-lactate: $\mathrm{H}^{+}$transport in wild-type tachyzoites. It is possible that the native $\operatorname{tg} f n t 2$ and $\operatorname{tg} f n t 3$ genes are not expressed at significant levels during this stage of the parasite life cycle.

Our $\mathrm{pH}$ data implicate TgFNT1 as the primary L-lactate transporter in tachyzoites. Erler et al. ${ }^{22}$ provided evidence for L-lactate transport by $T g F N T 1-3$ expressed in yeast, however truncation of the proteins was required to achieve their functional expression. To test whether full-length $T g F N T 1$ is a L-lactate transporter, we expressed it in Xenopus oocytes. We have previously expressed PfFNT in this system and shown that it transports L- $\left[{ }^{14} \mathrm{C}\right]$ lactate $^{15}$ and is inhibited by the antiplasmodial compound MMV007839 $9^{21}$. We measured the uptake of L- $\left[{ }^{14} \mathrm{C}\right]$ lactate into Xenopus oocytes expressing full-length $\mathrm{TgFNT} 1$ (with a C-terminal HA tag) at $27.5^{\circ} \mathrm{C}$ over $10 \mathrm{~min}$ at $\mathrm{pH}$ 6.4. The uptake of $\mathrm{L}_{-}\left[{ }^{14} \mathrm{C}\right]$ lactate by oocytes injected with $30 \mathrm{ng}$ of cRNA encoding $\mathrm{TgFNT} 1$ was higher than that of non-injected oocytes and of oocytes injected with 30 ng of cRNA encoding PfFNT $(P<0.001$; oneway ANOVA; Fig. 4). Consistent with previous results ${ }^{21}, \mathrm{~L}-\left[{ }^{14} \mathrm{C}\right]$ lactate uptake by PfFNT-expressing oocytes was inhibited by MMV007839 (4 $\mu \mathrm{M}$; Fig. 4). In contrast, $4 \mu \mathrm{M}$ MMV007839 had no effect on the uptake of $\mathrm{L}-\left[{ }^{14} \mathrm{C}\right]$ lactate by TgFNT1-expressing oocytes (Fig. 4). These data provide direct evidence that $T g \mathrm{FNT} 1$ is a L-lactate transporter. The finding that TgFNT1 is less sensitive than PfFNT to inhibition by MMV007839 is consistent with the results of Erler et al. ${ }^{22}$, who found that $29 \mu \mathrm{M}$ MMV007839 was required for half-maximal inhibition of L-lactate transport by a C-terminally truncated form of $\mathrm{TgFNT} 1$, whereas half-maximal inhibition of L-lactate transport by PfFNT is achieved at sub-micromolar concentrations ${ }^{20,21}$.

As a final test of the role of TgFNT1 in L-lactate transport across the plasma membrane in extracellular tachyzoites, we measured the uptake of $\mathrm{L}_{-}\left[{ }^{14} \mathrm{C}\right]$ lactate by extracellular $\Delta \operatorname{tg} f n t 1, \Delta \operatorname{tg} f n t 1 / 2 / 3$, and wild-type parental parasites. As noted above, FNTs mediate bidirectional transport, and measuring the movement of L- $\left[{ }^{14} \mathrm{C}\right]$ lactate into parasites provides a convenient means of assessing transport activity. Similar to previous experiments with P. falciparum ${ }^{15,21}$, the experiments with T. gondii presented here were conducted at a low $\mathrm{pH}(6.1)$ to increase $\left(\mathrm{H}^{+}\right.$-coupled) L-lactate uptake by the parasites, and at a low temperature $\left(4{ }^{\circ} \mathrm{C}\right)$ to slow the transport process. We found that the uptake of lactate, as measured over $10 \mathrm{~min}$, was greatly reduced in $\Delta \operatorname{tgfnt} 1$ and $\Delta \operatorname{tgfnt1/2/3}$ parasites relative to wild-type parental parasites, with little difference observed between $\Delta$ tgfnt 1 and $\Delta \operatorname{tgfnt1/2/3}$ parasites (Fig. 5a). We estimated the initial rate of L-lactate uptake for each parasite line. The initial rate of L-lactate uptake was $>7.5$-fold greater in wild-type parental parasites than in $\Delta \operatorname{tg} f n t 1$ or $\Delta \operatorname{tg} f n t 1 / 2 / 3$ parasites, and there was no significant difference in the initial rate between $\Delta \operatorname{tgfnt} 1$ and $\Delta \operatorname{tg} f n t 1 / 2 / 3$ parasites (Fig. 5b). Consistent with the $\mathrm{pH}$ data, these data indicate that $\mathrm{TgFNT} 1$ plays the primary role in L-lactate transport across the plasma membrane in wild-type extracellular parasites.

To investigate the possibility that disruption of the tgfnt1 gene could give rise to a general defect in solute uptake, we measured the uptake of two additional radiolabelled compounds: the glucose analogue $\left[{ }^{14} \mathrm{C}\right] 2$-deoxyglucose $\left(\left[{ }^{14} \mathrm{C}\right] 2-\mathrm{DOG}\right)$ and the amino acid $\mathrm{L}-\left[{ }^{14} \mathrm{C}\right]$ arginine. The time courses for the uptake of 
a



C

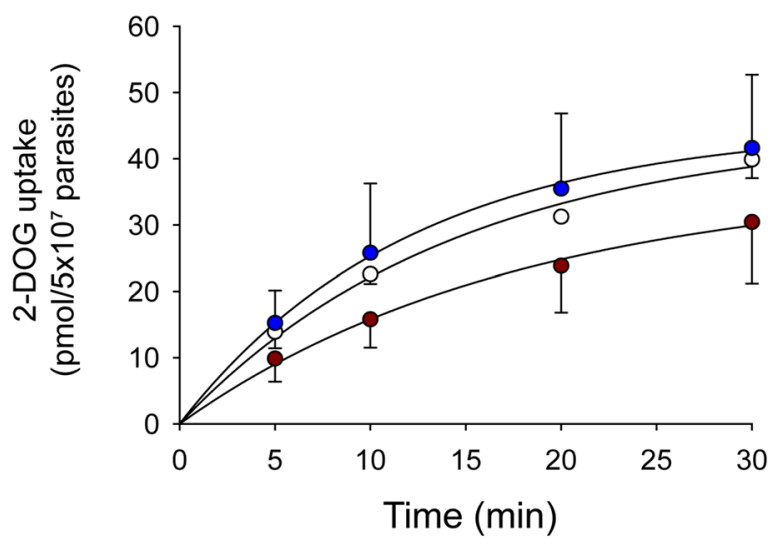

e

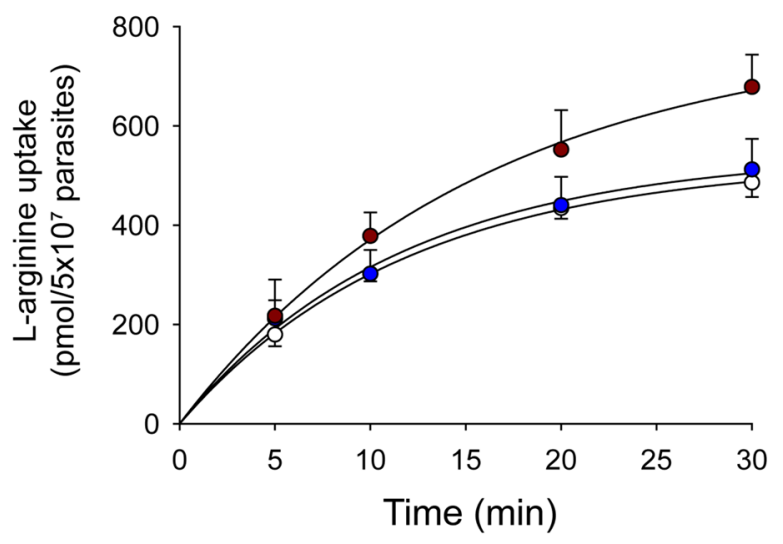

b



d
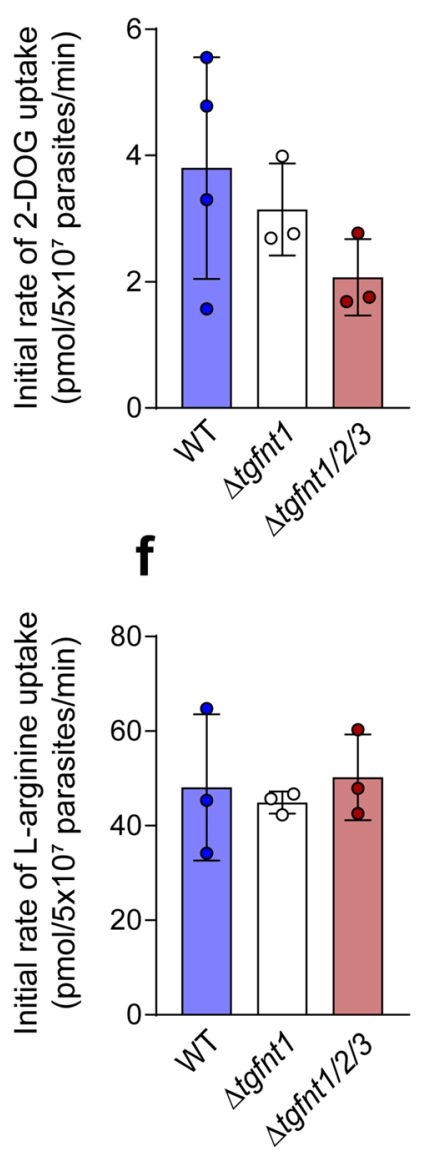

Figure 5. $T g F N T 1$ is critical for L-lactate transport across the parasite plasma membrane. (a,c,e) Time courses for the uptake of L-lactate (a), 2-DOG (c) and L-arginine (e) by wild-type parasites (blue symbols), $\Delta \operatorname{tg} f n t 1$ parasites (white symbols) and $\Delta t g f n t 1 / 2 / 3$ parasites (dark red symbols). The data shown are the mean \pm SD from three to four independent experiments for each line. For clarity, only positive or negative error bars are shown for the data for certain parasite lines. Where not shown, error bars fall within the symbols. (b,d,f) The initial rates of uptake for L-lactate (b), 2-DOG (d), and L-arginine (f) estimated from the same set of experiments. The bars show the mean \pm SD obtained from three or four independent experiments for each line, and the symbols show the results obtained in individual experiments. For panels $\mathbf{b}, \mathbf{d}$ and $\mathbf{f}$, the data for each parasite line were compared with the data for every other parasite line (one-way ANOVA, followed by post hoc Tukey tests where significant differences were present). ${ }^{* * * P}<0.001$; other comparisons (those in $\mathbf{d}$ and $\mathbf{f}$ ) did not reveal significant differences. 
2-DOG and L-arginine were similar in wild-type, $\Delta \operatorname{tg} f n t 1$ and $\Delta \operatorname{tg} f n t 1 / 2 / 3$ parasites (Fig. $5 c$,e), and there were no significant differences in the initial rates of uptake for either of these compounds between any of the parasite lines (Fig. 5 d,f). These data indicate that $\Delta \operatorname{tg} f n t 1$ and $\Delta t g f n t 1 / 2 / 3$ parasites do not have a general defect in solute uptake, but rather have a specific defect in the uptake of FNT substrates including L-lactate.

\section{Discussion}

In this study we pinpoint $\mathrm{TgFNT} 1$ as the primary contributor to L-lactate transport across the plasma membrane of extracellular T. gondii tachyzoites. Tagging the $3^{\prime}$ end of the native $\operatorname{tg} f n t 1$ gene revealed that the encoded protein localizes to the parasite plasma membrane. Experiments in which TgFNT1 was studied in isolation in Xenopus oocytes provided direct evidence that full-length $\mathrm{TgFNT} 1$ mediates the transport of $\mathrm{L}-\left[{ }^{14} \mathrm{C}\right]$ lactate. Using genetic and physiological approaches we confirmed that TgFNT1 functions as a plasma membrane L-lactate transporter in situ. We measured the rate of L-lactate transport across the parasite plasma membrane, and found that it was greatly reduced in $\Delta t g f n t 1$ tachyzoites compared to wild-type parasites. We also investigated the effect on cytosolic $\mathrm{pH}$ of adding $10 \mathrm{mM}$ L-lactate to the external medium. The decrease in cytosolic $\mathrm{pH}$ normally induced by a large inward L-lactate concentration gradient was not observed in $\Delta t g f n t 1$ parasites, consistent with these parasites having a defect in L-lactate: $\mathrm{H}^{+}$transport. Complementing $\Delta t g f n t 1$ parasites with an ectopic copy of the tgfnt1 gene restored the L-lactate-induced $\mathrm{pH}$ change.

Consistent with Erler et al. ${ }^{22}$, we found that TgFNT2 and TgFNT3 localize to the plasma membrane when expressed ectopically under the control of a non-native promoter. Furthermore, in line with previous evidence that truncated forms of TgFNT1-3 transport L-lactate in yeast ${ }^{22}$, our data suggest that (full-length) $T g$ FNT2 and $T g F N T 3$ can transport L-lactate. Complementing $\Delta \operatorname{tg} f n t 1$ parasites with constitutively-expressed $\operatorname{tg} f n t 2$ or $\operatorname{tg} f n t 3$ resulted in the restoration of $\mathrm{L}$-lactate: $\mathrm{H}^{+}$transport, as observed in measurements of cytosolic $\mathrm{pH}$.

Our data indicate that, despite being capable of transporting L-lactate, $T g$ FNT2 and TgFNT3 make little contribution to L-lactate transport across the plasma membrane in wild-type extracellular tachyzoites. Disrupting $\operatorname{tg} f n t 1$ alone was sufficient to abolish the L-lactate-induced acidification observed in parental parasites. Furthermore, there was no difference in the rate of $\mathrm{L}-\left[{ }^{14} \mathrm{C}\right]$ lactate transport across the plasma membrane in $\Delta \operatorname{tg} f n t 1$ parasites and $\Delta \operatorname{tgfnt1/2/3}$ parasites. Thus, expression of the endogenous $\operatorname{tg} f n t 2$ or $\operatorname{tg} f n t 3$ genes in $\Delta \operatorname{tg} f n t 1$ parasites does not appear to compensate for the L-lactate transport defect observed when TgFNT1 is not expressed. It is possible that $\mathrm{TgFNT} 2$ and $\mathrm{TgFNT} 3$ are not expressed at appreciable levels during the lytic cycle in the T. gondii strain used in this study. This would also explain our inability to localize these proteins by tagging the $3^{\prime}$ ends of the endogenous genes. Our data are partially supported by a recent proteome-wide localization study of tachyzoites, which predicted that $T g$ FNT1 localizes to the plasma membrane whereas TgFNT3 was not detectable in the proteome ${ }^{28}$. This study predicted that $\mathrm{TgFNT} 2$ may localize to micronemes, which is inconsistent with our localization studies.

A key finding in our study was that the lack of expression of all three $T g F N T$ proteins does not affect the proliferation of T. gondii tachyzoites under standard in vitro culture conditions. This contrasts with the situation in P. falciparum, where PfFNT has been validated as a drug target and must therefore be important for parasite proliferation ${ }^{20,21}$. However, it is consistent with previous studies highlighting the metabolic flexibility of T. gondii parasites, including recent evidence that the LDH enzymes required to convert pyruvate to lactate are not essential for T. gondii proliferation under standard in vitro culture conditions ${ }^{9,10}$. Our data are also consistent with a genome-wide screen that provided evidence that each of the TgFNTs are dispensable during the lytic cycle (the 'phenotype scores' for $T g$ FNT1, $T g$ FNT2 and $T g$ FNT3 were $1.10,1.31$ and -0.23 , respectively ${ }^{23}$ ), and with a very recent study in which TgFNT1 was knocked out ${ }^{24}$. Kloehn et al. ${ }^{24}$ found that TgFNT1 knockout parasites grew normally in vitro, but that they secreted less lactate into the medium than wild-type parasites. Our finding that all three $T g$ FNTs can be knocked out simultaneously call into question the view that the TgFNTs are suitable drug targets, and suggest that the compounds reported to inhibit the activity of $\mathrm{TgFNT}$ proteins by Erler et al. ${ }^{22}$ are unlikely to exert their effects on T. gondii growth via inhibition of any of the three transporters.

It should be noted that the disruption of certain glycolytic enzymes that are not essential for the in vitro proliferation of T. gondii parasites has been associated with virulence defects in mice and/or reductions in the formation of bradyzoite cysts in the brain. Parasites lacking hexokinase had a greatly reduced ability to form mature bradyzoite cysts in the brain in mice compared to parental parasites ${ }^{13}$. Furthermore, while tachyzoites in which the genes encoding $\mathrm{TgLDH} 1$ and $\mathrm{TgLDH} 2$ were simultaneously disrupted proliferated normally under standard conditions ${ }^{9,10}$, their growth was impaired in low-oxygen conditions in vitro ${ }^{10}$ and in mice ${ }^{9,10}$, and the numbers of bradyzoite cysts formed in the brain were greatly reduced ${ }^{9,10}$. Thus, it remains possible that the expression of one or more TgFNT proteins is important in vivo and/or in different stages of the T. gondii life cycle.

\section{Methods}

Ethics statement. Ethical approval of the work performed with the adult female Xenopus laevis frogs was obtained from the Australian National University (ANU) Animal Experimentation Ethics Committee (Animal Ethics Protocol Number A2013/13) in accordance with the Australian Code of Practice for the Care and Use of Animals for Scientific Purposes.

Host cell and parasite culture. T. gondii parasites were cultured in human foreskin fibroblasts (a gift from Holger Schlüter, Peter MacCallum Cancer Centre) at $37^{\circ} \mathrm{C}$ in a humidified $5 \% \mathrm{CO}_{2}$ incubator. The culture medium was Dulbecco's modified Eagle's medium (DMEM) containing $25 \mathrm{mM}$ glucose, and supplemented with $1 \% \mathrm{v} / \mathrm{v}$ fetal calf serum, $200 \mu \mathrm{M} \mathrm{L}$-glutamine, $50 \mathrm{U} / \mathrm{mL}$ penicillin, $50 \mu \mathrm{g} / \mathrm{mL}$ streptomycin, $10 \mu \mathrm{g} / \mathrm{mL}$ gentamicin, and $0.25 \mu \mathrm{g} / \mathrm{mL}$ amphotericin B. TATi/Tomato parasites ${ }^{29}$ were used as the parental wild-type strain for the 
$\Delta \operatorname{tg} f n t$ parasite strains that we generated in this study. TATi $\Delta k u 80$ parasites ${ }^{30}$ were used as the parental wild-type strain for the $3^{\prime}$ replacement strains generated in this study.

Generation of genetically modified T. gondii lines for localization studies. To generate a $3^{\prime}$ HA tag replacement in the $\operatorname{tg} f n t 1$ locus, we amplified a $1.1 \mathrm{~kb}$ region of the $3^{\prime}$ end of the $\operatorname{tg} f n t 1$ open reading frame using primers $\mathrm{p} 4$ and $\mathrm{p} 5$ (Supplementary Table S1). The PCR product was digested with BglII and AvrII and ligated into the $B g l \mathrm{II}$ and $A v r \mathrm{II}$ sites of the vector $\mathrm{pgCH}(\Delta P s t \mathrm{I})$, a variant of $\mathrm{pgCH}^{31}$ wherein the PstI site has been filled in. The resulting vector was linearized with $S p h \mathrm{I}$ and transfected into TATi/ $\Delta k u 80$ parasites using standard procedures $^{32}$, with successfully transfected parasites selected with chloramphenicol.

$T g$ FNT2 and $T g$ FNT3 were localized by expressing C-terminally $3 \times$ HA tagged forms of the proteins under the control of the constitutive $\alpha$-tubulin promoter. The $t g f n t 2$ open reading frame was amplified from $T$. gondii cDNA using primers p6 and p7 (Supplementary Table S1). The PCR product was digested with AvrII and BclI and ligated into the AvrII and BglII sites of the $\mathrm{pUgCTH} 3$ plasmid $^{31}$. The $\operatorname{tg} f n t 3$ open reading frame was amplified from cDNA using primers p8 and p9 (Supplementary Table S1). The PCR product was digested with AvrII and BglII and ligated into the AvrII and BglII sites of pUgCTH3. In both cases the vectors were transfected into TATi/tomato parasites ${ }^{29}$.

Generation and complementation of tgfnt knockout parasites. We used a CRISPR/Cas9-based genome editing approach to generate parasite lines in which the open reading frames of tgfnt genes were disrupted. Using the Q5 Site-Directed Mutagenesis kit (New England Biolabs), we generated modified versions of the pSAG1::Cas9-U6::sgUPRT vector (Addgene plasmid \# 54467 ${ }^{33}$ ) to express guide RNAs targeting each tgfnt gene using a Q5 mutagenesis approach, as described by the manufacturer (New England Biolabs). The primers used for the Q5 mutagenesis reactions were p10 and p11 for tgfnt1, p12 and p11 for tgfnt2, and p13 and p11 for tgfnt3 (Supplementary Table S1). The resulting vectors co-express the guide RNA targeting the appropriate $\operatorname{tg} f n t$ gene and Cas9-GFP. They were transfected into TATi/tomato parasites, generating $\Delta \operatorname{tg} f n t 1, \Delta \operatorname{tgfnt} 2$, and $\Delta \operatorname{tgfnt} 3$ parasites (Supplementary Fig. S4). Parasites in which two and three tgfnt genes were disrupted were then made by targeting the $\operatorname{tgfnt} 1$ gene in $\Delta t g f n t 3$ parasites then the $\operatorname{tg} f n t 2$ gene in $\Delta \operatorname{tg} f n t 1 / 3$ parasites (Supplementary Fig. S4).

For complementation studies, $\Delta t g f n t 1$ parasites were transfected with vectors containing the $\operatorname{tg} f n t 1$, tgfnt2 or tgfnt 3 gene fused at the $3^{\prime}$ end to a $3 \times \mathrm{HA}$ tag, under the control of the constitutive $\alpha$-tubulin promoter. For $T g$ FNT2 and $T g$ FNT3, the vectors used were those described above for the localization of the ectopically expressed proteins. The complementation vector for $T g$ FNT1 was made by amplifying the tgfnt 1 open reading frame from genomic DNA using primers p14 and p5 (Supplementary Table S1). The PCR product was digested with AvrII and BglII and ligated into the AvrII and BglII sites of pUgCTH3.

Immunofluorescence assays and western blotting. Immunofluorescence assays (IFAs) and western blots were carried out as described previously ${ }^{25}$. The primary antibodies used were rat anti-HA (Roche clone 3F10; 1:100 - 1:1000 dilution; used for IFAs and western blots) and mouse anti-P30 (Abcam clone TP3; 1:500 dilution; used for IFAs). The secondary antibodies used for IFAs (all from Life Technologies) were goat anti-rat AlexaFluor 488 (1:100 - 1:250 dilution), goat anti-mouse AlexaFluor 546 (1:500 dilution), and goat anti-mouse AlexaFluor 647 (1:500 dilution). The microscopy was performed as described previously ${ }^{26}$. For western blots the secondary antibody was horse radish peroxidase-conjugated goat anti-rat (Santa Cruz Biotechnology; 1:5000 dilution).

Parasite growth assays. Parasite growth was measured in the culture medium described above, which contains $25 \mathrm{mM}$ glucose. Plaque assays were performed essentially as described previously $\mathrm{y}^{32}$, with 400 parasites added per $25 \mathrm{~cm}^{2}$ flask. Fluorescence growth assays were performed in 96 -well plates ${ }^{25,34}$, with 4000 parasites added to each well and the fluorescence from tdTomato (excitation: $540 \mathrm{~nm}$; emission: $590 \mathrm{~nm}$ ) used to monitor parasite growth. Prior to estimating parasite growth rates, a value corresponding to the background fluorescence (averaged from wells to which parasites had not been added) was subtracted from all other data. The fluorescence intensity corresponding to maximum growth was then determined for each parasite line by fitting a sigmoidal curve to the data: $y=a /\left[1+\left(t / t_{1 / 2}\right)^{b}\right]$, where $y$ is fluorescence intensity, $a$ is the maximum fluorescence intensity, $t$ is time and $t_{1 / 2}$ is the time at which half-maximal growth is reached. The data for each parasite line were then expressed as a $\%$ of the maximum growth for that line. For each parasite line, the maximum growth rate was determined from the slope of the sigmoidal curve in a $4 \mathrm{~h}$ window surrounding the $t_{1 / 2}$ (estimated by fitting the sigmoidal curve described above).

pH measurements in extracellular tachyzoites. Parasites were harvested by passage of cultures through a $26 \mathrm{G}$ needle. The parasites were then passed through a $3 \mu \mathrm{m}$ filter to remove host cell debris and centrifuged for $20 \mathrm{~min}$ at $1500 \times \mathrm{g}$. The supernatant media was removed and the parasites were suspended in 'BCF' (bicarbonate-free RPMI supplemented with $25 \mathrm{mM}$ HEPES, $11 \mathrm{mM}$ additional glucose, $0.2 \mathrm{mM}$ hypoxanthine and $\mathrm{pH}$-adjusted to 7.1 ) then centrifuged at $12,000 \times g$ for $1 \mathrm{~min}$. The supernatant media was removed and the parasites suspended again in BCF, to which the acetoxymethyl ester (AM) form of the $\mathrm{pH}$-sensitive dye BCECF (Molecular Probes) was added at a final concentration of $5 \mu \mathrm{M}$. The parasites were incubated in the BCECF-containing media for 14 min before being centrifuged $(12,000 \times g, 1 \mathrm{~min})$, resuspended in BCF and maintained at $37^{\circ} \mathrm{C}$. Directly before their use in fluorescence measurements, parasites were centrifuged $(12,000 \times g, 1 \mathrm{~min})$ and resuspended in $\mathrm{pH} 7.1$ Saline Solution $(125 \mathrm{mM} \mathrm{NaCl}, 5 \mathrm{mM} \mathrm{KCl}, 25 \mathrm{mM}$ HEPES, $20 \mathrm{mM}$ glucose and $1 \mathrm{mM}$ $\mathrm{MgCl}_{2}$ ). Fluorescence measurements were performed at $4{ }^{\circ} \mathrm{C}$ in a Cary Eclipse Fluorescence Spectrophotometer 
(Agilent). The excitation wavelengths were $440 \mathrm{~nm}$ and $495 \mathrm{~nm}$ and the emission wavelength was $520 \mathrm{~nm}$. The ratio of fluorescence ( $495 \mathrm{~nm} / 440 \mathrm{~nm}$ ) was used as an indicator of cytosolic $\mathrm{pH}$. The relationship between Fluorescence Ratio and $\mathrm{pH}$ was determined by suspending parasites in calibration salines of known $\mathrm{pH}^{35}$ containing $16 \mu \mathrm{M}$ nigericin (to equilibrate $\mathrm{H}^{+}$across the plasma membrane) and measuring the Fluorescence Ratio after it stabilized.

Measurements of the uptake of radiolabelled compounds by parasites. The uptake of radiolabelled compounds was measured in extracellular tachyzoites suspended in $\mathrm{pH} 6.1$ Saline Solution (for L- $\left[{ }^{14} \mathrm{C}\right]$ lactate and $\mathrm{L}-\left[{ }^{14} \mathrm{C}\right]$ arginine experiments) or Glucose-free Saline Solution (for $\left[{ }^{14} \mathrm{C}\right] 2$-DOG experiments; $125 \mathrm{mM}$ $\mathrm{NaCl}, 5 \mathrm{mM} \mathrm{KCl}, 25 \mathrm{mM}$ HEPES, $1 \mathrm{mM} \mathrm{MgCl}_{2} ; \mathrm{pH}$ 6.1). The reactions for L-lactate experiments contained $200 \mu \mathrm{M}$ unlabelled L-lactate and $0.2 \mu \mathrm{Ci} / \mathrm{mL} \mathrm{L}^{-}\left[{ }^{14} \mathrm{C}\right]$ lactate, those for the 2-DOG experiments contained $25 \mu \mathrm{M}$ unlabelled 2-DOG and $0.2 \mu \mathrm{Ci} / \mathrm{mL}\left[{ }^{14} \mathrm{C}\right] 2-\mathrm{DOG}$, and those for the $\mathrm{L}$-arginine experiments contained $50 \mu \mathrm{M}$ unlabelled L-arginine and $0.1 \mu \mathrm{Ci} / \mathrm{mL} \mathrm{L}-\left[{ }^{14} \mathrm{C}\right]$ arginine.

The $\mathrm{L}-\left[{ }^{14} \mathrm{C}\right]$ lactate and $\left[{ }^{14} \mathrm{C}\right] 2-\mathrm{DOG}$ experiments were conducted on ice, with centrifugation steps performed at $4{ }^{\circ} \mathrm{C}$, to slow the rate of uptake such that initial rates could be determined. For the $\mathrm{L}_{-}\left[{ }^{14} \mathrm{C}\right]$ arginine experiments, parasites were incubated on ice for $30 \mathrm{~min}$ prior to commencing the experiments to mimic exposure conditions for the $\mathrm{L}-\left[{ }^{14} \mathrm{C}\right]$ lactate experiments, but the experiments themselves were performed at $37^{\circ} \mathrm{C}$.

Uptake assays were performed as described previously ${ }^{36}$. To commence an experiment, a volume of parasite suspension was added to an equal volume of a saline solution containing the radiolabelled compound. The uptake of radiolabelled compounds was stopped at pre-determined time points (in duplicate $200 \mu \mathrm{L}$ samples) by centrifuging the samples in tubes containing $250 \mu \mathrm{L}$ oil mix (84\% Clerco PM-125 silicone fluid, $16 \%$ light mineral oil), thereby sedimenting the parasites below the oil layer. Aliquots $(10 \mu \mathrm{L})$ of the supernatant solution were taken from above the oil layer to enable the extracellular concentrations of the radiolabelled compounds to be determined. For each tube, the remaining supernatant solution was removed, the tubes washed twice in running water, the oil aspirated, and the parasite pellet lysed with $500 \mu \mathrm{L} 0.1 \% \mathrm{v} / \mathrm{v}$ Triton X-100. The lysate was transferred into a scintillation vial containing $1.5 \mathrm{~mL}$ scintillation fluid (Ultima Gold; Perkin Elmer), and the radioactivity measured using a scintillation counter.

To account for the radioactivity present in the extracellular fluid trapped around the cell pellet under the oil layer, we subtracted the amount of radioactivity present at time zero from the remaining data. Where there was significant uptake of radioactivity early in the time course $\left(\mathrm{L}-\left[{ }^{14} \mathrm{C}\right]\right.$ lactate and $\mathrm{L}-\left[{ }^{14} \mathrm{C}\right]$ arginine experiments $)$, we fit a curve to the time course data for each experiment $\left[y=y_{0}+a\left(1-\mathrm{e}^{(-\mathrm{b} x)}\right)\right]$ to determine the amount of radioactivity present at time zero $\left(y_{0}\right)$. For $\left[{ }^{14} \mathrm{C}\right] 2$-DOG experiments the radioactivity measured at the first time point (taken within $30 \mathrm{~s}$ of exposure of parasites to $\left.\left[{ }^{14} \mathrm{C}\right] 2-\mathrm{DOG}\right)$ was subtracted directly. A curve $\left[y=a\left(1-\mathrm{e}^{(-\mathrm{b} x)}\right)\right]$ was fitted to the data for intracellular radioactivity versus time and the initial rates were determined from the first derivative.

Experiments with Xenopus laevis oocytes. The frogs were purchased from Nasco (Cat\# LM00535M) and were housed in the Xenopus Frog Facility of the ANU Research School of Biology in compliance with the relevant institutional and Australian Government regulations.

The oocyte expression vector containing $p f f n t$ was made previously ${ }^{15}$. The oocyte expression vector containing $\operatorname{tg} f n t 1$ was made by amplifying the $t g f n t 1$ open reading frame from gDNA using primers p14 and p5 (Supplementary Table S1). The PCR product was digested with AvrII and XmaI and ligated into the AvrII and XmaI sites of $\mathrm{pGHJ}-\mathrm{HA}^{31}$.

Oocytes were harvested from $X$. laevis frogs and prepared as outlined previously ${ }^{37}$. cRNA encoding PfFNT and TgFNT1-HA was made using the mMessage mMachine T7 transcription kit and the MEGAclear kit (Ambion) and $30 \mathrm{ng}$ was microinjected into oocytes, as described elsewhere ${ }^{37}$. The uptake of $\mathrm{L}_{-}\left[{ }^{14} \mathrm{C}\right] \mathrm{lactic}$ acid $\left(\mathrm{Na}^{+}\right.$salt; $150.6 \mathrm{mCi} / \mathrm{mmol}$; Perkin Elmer) was measured 3-4 days post-injection at $27.5^{\circ} \mathrm{C}$ in ND96 buffer $(96 \mathrm{mM} \mathrm{NaCl}$, $2 \mathrm{mM} \mathrm{KCl}, 1 \mathrm{mM} \mathrm{MgCl}, 1.8 \mathrm{mM} \mathrm{CaCl}_{2}, 10 \mathrm{mM}$ MES and $10 \mathrm{mM}$ Tris-base; $\mathrm{pH}$ 6.4). Within each experiment measurements were made from 7 to 10 oocytes per condition. The influx of $\mathrm{L}-\left[{ }^{14} \mathrm{C}\right]$ lactate was terminated by removing the reaction buffer and washing the oocytes twice in $3.5 \mathrm{~mL}$ of ice-cold ND96 buffer. The oocytes were then lysed and the radioactivity measured as described elsewhere ${ }^{38}$.

Received: 24 August 2020; Accepted: 10 March 2021

Published online: 24 March 2021

\section{References}

1. W.H.O. World malaria report. (2018)

2. Blader, I. J., Coleman, B. I., Chen, C. T. \& Gubbels, M. J. Lytic cycle of Toxoplasma gondii: 15 years later. Annu. Rev. Microbiol. 69, 463-485 (2015).

3. Blume, M. et al. Host-derived glucose and its transporter in the obligate intracellular pathogen Toxoplasma gondii are dispensable by glutaminolysis. Proc. Natl Acad. Sci. USA 106, 12998-13003 (2009).

4. Nitzsche, R., Zagoriy, V., Lucius, R. \& Gupta, N. Metabolic cooperation of glucose and glutamine is essential for the lytic cycle of obligate intracellular parasite Toxoplasma gondii. J. Biol. Chem. 291, 126-141 (2016).

5. Xia, N. et al. Pyruvate homeostasis as a determinant of parasite growth and metabolic plasticity in Toxoplasma gondii. mBio 10, e00898-19 (2019).

6. Hayward, J. A. \& van Dooren, G. G. Same same, but different: Uncovering unique features of the mitochondrial respiratory chain of apicomplexans. Mol. Biochem. Parasitol. 232, 111204 (2019).

7. MacRae, J. I. et al. Mitochondrial metabolism of glucose and glutamine is required for intracellular growth of Toxoplasma gondii. Cell Host Microbe 12, 682-692 (2012). 
8. Ohsaka, A., Yoshikawa, K. \& Hagiwara, T. ${ }^{1} \mathrm{H}-\mathrm{NMR}$ spectroscopic study of aerobic glucose metabolism in Toxoplasma gondii harvested from the peritoneal exudate of experimentally infected mice. Physiol. Chem. Phys. 14, 381-384 (1982).

9. Abdelbaset, A. E. et al. Lactate dehydrogenase in Toxoplasma gondii controls virulence, bradyzoite differentiation, and chronic infection. PLoS ONE 12, e0173745 (2017).

10. Xia, N. et al. Functional analysis of Toxoplasma lactate dehydrogenases suggests critical roles of lactate fermentation for parasite growth in vivo. Cell. Microbiol. 20, e12794 (2018).

11. Nitzsche, R., Gunay-Esiyok, O., Tischer, M., Zagoriy, V. \& Gupta, N. A plant/fungal-type phosphoenolpyruvate carboxykinase located in the parasite mitochondrion ensures glucose-independent survival of Toxoplasma gondii. J. Biol. Chem. 292, 15225-15239 (2017).

12. Shen, B. \& Sibley, L. D. Toxoplasma aldolase is required for metabolism but dispensable for host-cell invasion. Proc. Natl Acad. Sci. USA 111, 3567-3572 (2014).

13. Shukla, A. et al. Glycolysis is important for optimal asexual growth and formation of mature tissue cysts by Toxoplasma gondii. Int. J. Parasitol. 48, 955-968 (2018).

14. MacRae, J. I. et al. Mitochondrial metabolism of sexual and asexual blood stages of the malaria parasite Plasmodium falciparum. BMC Biol. 11, 67 (2013).

15. Marchetti, R. V. et al. A lactate and formate transporter in the intraerythrocytic malaria parasite, Plasmodium falciparum. Nat. Commun. 6, 6721 (2015).

16. Wu, B. et al. Identity of a Plasmodium lactate/ $\mathrm{H}^{+}$symporter structurally unrelated to human transporters. Nat. Commun. 6, 6284 (2015).

17. Czyzewski, B. K. \& Wang, D. N. Identification and characterization of a bacterial hydrosulphide ion channel. Nature 483, 494-497 (2012).

18. Jia, W., Tovell, N., Clegg, S., Trimmer, M. \& Cole, J. A single channel for nitrate uptake, nitrite export and nitrite uptake by Escherichia coli NarU and a role for NirC in nitrite export and uptake. Biochem. J. 417, 297-304 (2009).

19. Suppmann, B. \& Sawers, G. Isolation and characterization of hypophosphite-resistant mutants of Escherichia coli: Identification of the FocA protein, encoded by the pfl operon, as a putative formate transporter. Mol. Microbiol. 11, 965-982 (1994).

20. Golldack, A. et al. Substrate-analogous inhibitors exert antimalarial action by targeting the Plasmodium lactate transporter PfFNT at nanomolar scale. PLoS Pathog. 13, e1006172 (2017).

21. Hapuarachchi, S. V. et al. The malaria parasite's lactate transporter PfFNT is the target of antiplasmodial compounds identified in whole cell phenotypic screens. PLoS Pathog. 13, e1006180 (2017).

22. Erler, H., Ren, B., Gupta, N. \& Beitz, E. The intracellular parasite Toxoplasma gondii harbors three druggable FNT-type formate and L-lactate transporters in the plasma membrane. J. Biol. Chem. 293, 17622-17630 (2018).

23. Sidik, S. M. et al. A genome-wide CRISPR screen in Toxoplasma identifies essential apicomplexan genes. Cell 166, 1423-1435 (2016).

24. Kloehn, J. et al. Multi-omics analysis delineates the distinct functions of sub-cellular acetyl-CoA pools in Toxoplasma gondii. BMC Biol. 18, 67 (2020).

25. van Dooren, G. G., Tomova, C., Agrawal, S., Humbel, B. M. \& Striepen, B. Toxoplasma gondii Tic20 is essential for apicoplast protein import. Proc. Natl Acad. Sci. USA 105, 13574-13579 (2008).

26. Lehane, A. M. et al. Characterization of the ATP4 ion pump in Toxoplasma gondii. J. Biol. Chem. 294, 5720-5734 (2019).

27. Moreno, S. N., Zhong, L., Lu, H. G., Souza, W. D. \& Benchimol, M. Vacuolar-type H ${ }^{+}$-ATPase regulates cytoplasmic pH in Toxoplasma gondii tachyzoites. Biochem. J. 330, 853-860 (1998).

28. Barylyuk, K. et al. A comprehensive subcellular atlas of the Toxoplasma proteome via hyperLOPIT provides spatial context for protein functions. Cell Host Microbe 28, 752-766.e9 (2020).

29. Parker, K. E. R. et al. The tyrosine transporter of Toxoplasma gondii is a member of the newly defined apicomplexan amino acid transporter (ApiAT) family. PLoS Pathog. 15, e1007577 (2019).

30. Sheiner, L. et al. A systematic screen to discover and analyze apicoplast proteins identifies a conserved and essential protein import factor. PLoS Pathog. 7, e1002392 (2011).

31. Rajendran, E. et al. Cationic amino acid transporters play key roles in the survival and transmission of apicomplexan parasites. Nat. Commun. 8, 14455 (2017).

32. Striepen, B. \& Soldati, D. Genetic manipulation of Toxoplasma gondii. In Toxoplasma gondii: The Model Apicomplexan - Perspectives and Methods. (Weiss, L. D., and Kim, K. eds.) 391-415 (Elsevier, 2007).

33. Shen, B., Brown, K. M., Lee, T. D. \& Sibley, L. D. Efficient gene disruption in diverse strains of Toxoplasma gondii using CRISPR/ CAS9. mBio 5, e01114-14 (2014).

34. Gubbels, M. J., Li, C. \& Striepen, B. High-throughput growth assay for Toxoplasma gondii using yellow fluorescent protein. Antimicrob. Agents Chemother. 47, 309-316 (2003).

35. Saliba, K. J. \& Kirk, K. pH regulation in the intracellular malaria parasite, Plasmodium falciparum. $\mathrm{H}^{+}$extrusion via a V-type $\mathrm{H}^{+}$-ATPase. J. Biol. Chem. 274, 33213-33219 (1999).

36. Rajendran, E., Kirk, K. \& van Dooren, G. G. Measuring solute transport in Toxoplasma gondii parasites. Methods Mol. Biol. 2071, 245-268 (2020).

37. van Schalkwyk, D. A. et al. Verapamil-sensitive transport of quinacrine and methylene blue via the Plasmodium falciparum Chloroquine Resistance Transporter reduces the parasite's susceptibility to these tricyclic drugs. J. Infect. Dis. 213, 800-810 (2016).

38. Richards, S. N. et al. Molecular mechanisms for drug hypersensitivity induced by the malaria parasite's Chloroquine Resistance Transporter. PLoS Pathog. 12, e1005725 (2016).

39. Sibley, L. D., Krahenbuhl, J. L., Adams, G. M. \& Weidner, E. Toxoplasma modifies macrophage phagosomes by secretion of a vesicular network rich in surface proteins. J. Cell Biol. 103, 867-874 (1986).

\section{Acknowledgements}

This work was supported by an Australian Research Council (ARC) Discovery Early Career Researcher Award (DE160101035 to A.M.L.), ARC Discovery Grants (DP150102883 and DP200100483 to K.K. and G.G.v.D.), an ARC Future Fellowship (1053082 to R.E.M.), and the Australian Department of Education (Australian Postgraduate Awards to S.V.H. and S.H.S.).

\section{Author contributions}

J.M.Z., K.K., G.G.v.D. and A.M.L. designed the study. J.M.Z, S.V.H. and S.H.S. performed experiments. J.M.Z., S.V.H., S.H.S., R.E.M., G.G.v.D. and A.M.L. analysed data. R.E.M., K.K., G.G.v.D. and A.M.L. supervised the study and provided resources. J.M.Z., G.G.v.D. and A.M.L. wrote the manuscript. All authors reviewed the manuscript.

\section{Competing interests}

The authors declare no competing interests. 


\section{Additional information}

Supplementary Information The online version contains supplementary material available at https:/doi.org/ 10.1038/s41598-021-86204-3.

Correspondence and requests for materials should be addressed to G.G.v.D. or A.M.L.

Reprints and permissions information is available at www.nature.com/reprints.

Publisher's note Springer Nature remains neutral with regard to jurisdictional claims in published maps and institutional affiliations.

(c) (i) Open Access This article is licensed under a Creative Commons Attribution 4.0 International License, which permits use, sharing, adaptation, distribution and reproduction in any medium or format, as long as you give appropriate credit to the original author(s) and the source, provide a link to the Creative Commons licence, and indicate if changes were made. The images or other third party material in this article are included in the article's Creative Commons licence, unless indicated otherwise in a credit line to the material. If material is not included in the article's Creative Commons licence and your intended use is not permitted by statutory regulation or exceeds the permitted use, you will need to obtain permission directly from the copyright holder. To view a copy of this licence, visit http://creativecommons.org/licenses/by/4.0/.

(C) The Author(s) 2021 